\section{P84 THE INSPIWAVE (TM) TRIAL ON ADULT HEALTHY VOLUNTEERS - INSIGHTS GLEANED FROM POSTURAL STUDIES}

${ }^{1} \mathrm{C}$ Zhang, ${ }^{2} \mathrm{P}$ Phan, ${ }^{3} \mathrm{D}$ Geer, ${ }^{2} \mathrm{C}$ Hahn, ${ }^{2} \mathrm{~A}$ Farmery. ${ }^{1} \mathrm{G} K T$ School of Medical Education, King's College London, London, UK; ${ }^{2}$ Nuffield Department of Clinical Neurosciences, University of Oxford, Oxford, UK; ${ }^{3}$ The Queen's College, University of Oxford, Oxford, UK

\subsection{6/thoraxjnl-2015-207770.221}

Introduction Current techniques used to monitor Functional Residual Capacity (FRC) present significant clinical limitations which often restrict patients to those who are conscious, cooperative and mobile. There is a clinical need for an operator - independent device that is able to simultaneously and continuously monitor FRC in mechanically ventilated patients, the morbidly obese and infants. The InspiWave ${ }^{\mathrm{TM}}$ is one such device which utilises a sinusoidal Nitrous Oxide $\left(\mathrm{N}_{2} \mathrm{O}\right)$ tracer gas technique to non - invasively monitor various parameters of cardiopulmonary function, including end tidal alveolar volume $\left(\mathrm{V}_{\mathrm{A}}\right)$ and $\mathrm{Bohr}$
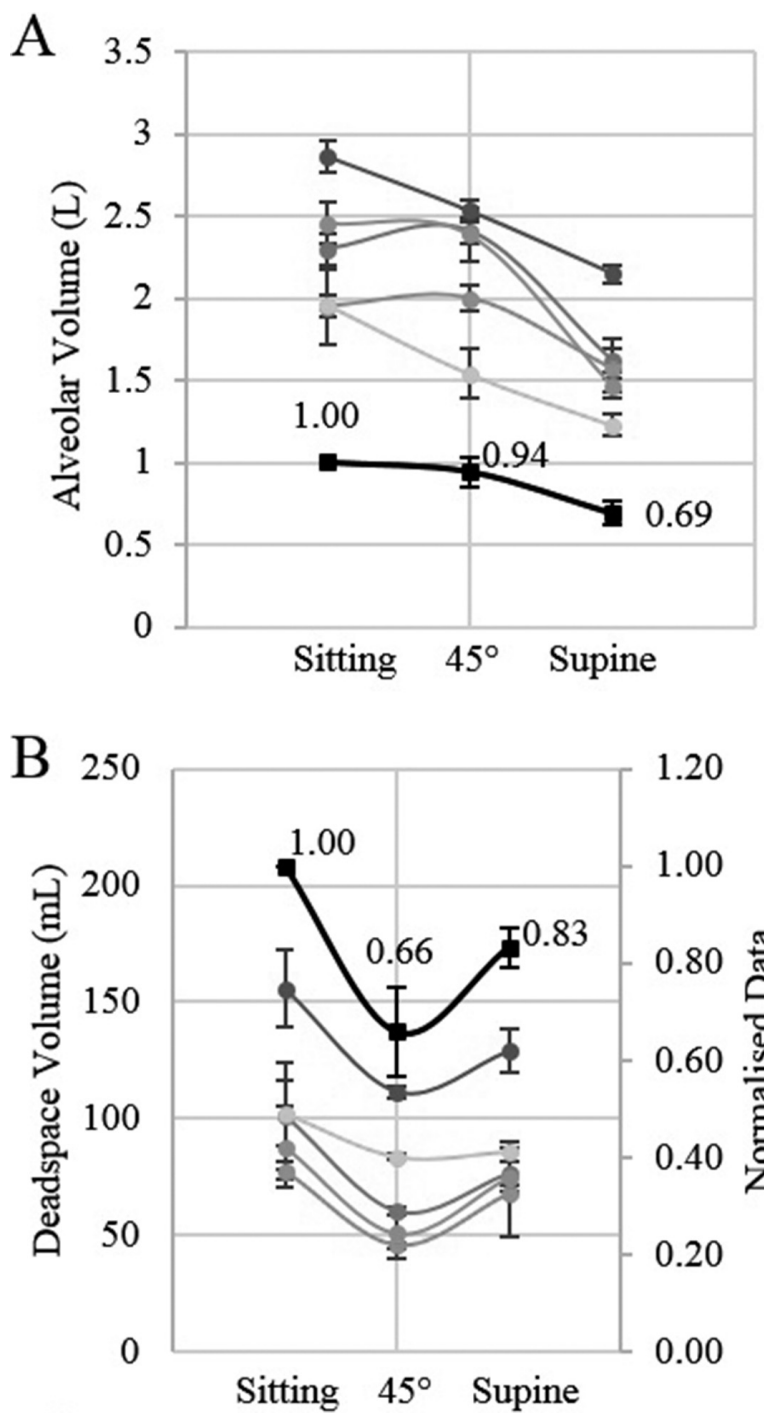

1.20

1.00

0.80

0.60

0.40

0.20

0.00

Abstract P84 Figure 1 Alveolar Volume (A) and Deadspace Volume (B) estimated by the device at three different postures. Averaged data of participants $(n=5)$ shown in traces with circle markers $(\bullet)$, error bars represent standard deviation. Normalised data relative to the upright sitting position plotted with square markers (ם), error bars represent $95 \%$ confidence interval deadspace volume $\left(\mathrm{V}_{\mathrm{D}}\right)$. This study evaluates the performance of the InspiWave ${ }^{\mathrm{TM}}$ in estimating lung volume using two unique postural change protocols in healthy volunteers.

Method 5 subjects were asked to adopt 3 stationary postures (sitting upright, supine, $45^{\circ}$ elevation) using a tilt table. 21 subjects adopted a different protocol, with a single transition phase between sitting and supine.

Results In the first protocol $(n=5), V_{A}$ estimations were consistently smaller at supine than at sitting (31\%). At $45^{\circ}$ elevation, $V_{D}$ estimations were the smallest of all three postures. Comparison of absolute flow signal $\left(\Delta \int\right.$ Flow $\left.d t\right)$ data at the point of postural transition with $\mathrm{V}_{\mathrm{A}}$ estimations by the device $(\mathrm{n}=21)$ yielded a Pearson's correlation $(r)$ of $0.453(p<0.05)$.

Conclusion Estimations by the InspiWave ${ }^{\mathrm{TM}}$ demonstrated acceptable correlation between absolute flow signal change and device estimation, suggesting good accuracy. FRC changes by approximately $25 \%$ from sitting to supine, due to added pressure from abdominal contents on the diaphragm when gravity shifts with postural change. ${ }^{1}$ Data from this study closely mirrored this value (31\%), demonstrating a good degree of device performance. $V_{D}$ estimations were smallest at $45^{\circ}$ elevation of the three postures which may be due to airways being kept more open, consistent with respiratory benefits of the $45^{\circ}$ "beach chair position”. Postural studies provide an alternative over conventional comparative studies with reference techniques (such as body plethysmography) in assessing device performance, when comparing variables which are not entirely equivalent.

\section{REFERENCE}

1 Ibanez J, Raurich JM. Normal values of functional residual capacity in the sitting and supine positions. Intensive Care Med. 1982;8(4):173-177

\section{P85 A COMPOSITE INDEX OF SATURATION AND DISTANCE WALKED DURING A 6-MINUTE WALK TEST (6MWT): A RETROSPECTIVE METHODOLOGICAL COMPARISON}

R Brown, AH Kendrick. Department of Respiratory Medicine, University Hospitals, Bristol, UK 10.1136/thoraxjnl-2015-207770.222

Background The 6MWT is a simple, reproducible test of exercise performance. Lettieri et al. $^{1}$ proposed a composite index of the product of the lowest oxygen saturation $\left(\mathrm{SpO}_{2}\right)$ and distanced walked - the distance-saturation product (DSP). Pimenta et al. ${ }^{2}$ calculated the differences between actual $\mathrm{SpO}_{2}$ and $100 \%$ every two seconds, and produced the desaturation-distance ratio (DDR-1). Ijiri et al. ${ }^{3}$ simplified Pimenta's methodology, using the $\mathrm{SpO}_{2}$ at the end of each minute (DDR-2).

Aim To compare the different methods of calculating the composite index.

Methods We retrospectively analysed 48 6MWT datasets, obtained using standard methodology. $\mathrm{SpO}_{2}$ was recorded every second using a Minolta 300i pulse oximeter and analysed using Visi-Download (Stowood Scientific, Oxford). The DSP and DDR's was calculated by each method. Additionally, data using Pimenta's method, was calculated using one second data intervals (DDR-3). Data are given as median (IQR).

Results $41 / 48$ patients were male. Group age was 63 (53 to 73) years, $\mathrm{FEV}_{1}$ \%pred $68.4 \%$ (41.3 to 82.3 ), distance walked $390 \mathrm{~m}$ (321.3 to 477.5), baseline $\mathrm{SpO}_{2}$ 95\% (93 to 96) and decline in $\mathrm{SpO}_{2}$ was $-4 \%$ (-2 to -8$)$. DSP was 324.6 (278.8 to 419.4$) \mathrm{m} \%$, DDR-1 was 8.90 (4.98 to 13.27), DDR-2 was 16.83 (9.14 to 24.8) and DDR-3 was 8.90 (4.99 to 13.66). Bland-Altman 
analysis of DDR-1 vs DDR-3 gave a bias of -0.0083 and $95 \%$ limits of -0.042 to 0.025 . Correlations between each index and $\mathrm{FEV}_{1}$ \%pred, $\mathrm{SpO}_{2}$ and Borg were significant for $\mathrm{FEV}_{1}$ \%pred and $\mathrm{SpO}_{2}$, but not significant for Borg (Table 1).

\begin{tabular}{|c|c|c|c|}
\hline & $\mathrm{FEV}_{1} \%$ pred & $\Delta \mathrm{SpO}_{2}$ & Borg \\
\hline DSP & 0.53 & 0.1612 & 0.046 \\
\hline DDR-1 & 0.71 & 0.2246 & 0.034 \\
\hline DDR-2 & 0.72 & 0.2268 & 0.033 \\
\hline DDR-3 & 0.71 & 0.2251 & 0.035 \\
\hline
\end{tabular}

Conclusions 1) there is no significant difference between DDR-1 and DDR-3, both providing an accurate assessment of changes in $\mathrm{SpO}_{2}$ during exercise and allowing for the different storage capabilities of pulse oximeters; 2) the simplest index (DSP), showed poorer correlations cpmpared to the DDR's, perhaps reflecting the simplicity of the index; 3 ) the conceptual idea of a composite index of distance walked and changes in $\mathrm{SpO}_{2}$ during a 6MWT needs further investigation in a range of different clinical settings.

\section{REFERENCES}

1 Lettieri CJ, Nathan SD, Browning RF, et al. The distance-saturation product predicts mortality in idiopathic pulmonary fibrosis. Respir Med. 2006;100: 1734-1741

2 Pimenta SP, Rocha RB, Baldi BG, et al. Clinics 2010;65:841-846

3 Ijiri N, Kanazawa H, Yoshikawa T, Hirata K, et al. Application of a new parameter in the 6-minute walk test for manifold analysis of exercise capacity in patients with COPD. Int J Chron Obstruct Pulmon Dis. 2014;9:1235-1240

\section{P86 SPIROMETRIC VALUES OF GREEK HEALTHY PEOPLE AND COMPARISON WITH ECSC VALUES IN COPD PEOPLE}

${ }^{1} \mathrm{~N}$ Tatsis, ${ }^{1} \mathrm{~S}$ Kakavas, ${ }^{1} \mathrm{E}$ Balis, ${ }^{2} \mathrm{~N}$ Koulouris, ${ }^{2} \mathrm{~K}$ Hadjistavrou, ${ }^{1} \mathrm{G}$ Tatsis. ${ }^{1}$ Pulmonary Department of Evangelismos General Hospital, Athens, Greece; ${ }^{2}$ University Pulmonary Clinic of Sotiria Hospital, Athens, Greece

\subsection{6/thoraxjn-2015-207770.223}

Introduction There have been always controversies whether, there is a hypo or hyper diagnosis of COPD according GOLD criteria with parameters driven many years ago, around many countries.

Purpose The purpose of our study is the use of mathematical models for the comparison of Greek patients with COPD, according GOLD criteria versus normal values of our country.

Methods Using spirometry results from a pool of healthy local population ( $\mathrm{n}=500$, age range $18-89$ years) we fitted regression models, separately for men and women, for FEV1 and FEV1/FVC ratio. The set of healthy individuals consisted of 261 women (52.2\%) and 239 men (47.8\%). Their mean age (SD) was $48.20 \pm 17.19$ and $46.92 \pm 16.16$ years respectively. The corresponding heights were $162.7 \pm 7.2$ and175.8 $\pm 7.3 \mathrm{~cm}$. Predicted normal FEV1 was also calculated using the European Coal and Steel Community (ECSC) equations. Also 124 subjects, with a history of COPD were studied (age range 25-91 years). Results A positive and linear association with height was apparent in women whereas for men, a guadratic height term was also statistically significant. Predicted FEV1 of healthy individuals, based on either the ECSC or the locally derived equations, was excellent with the concordance correlation coefficients being 0.986 for women and 0.991 for men $(p<0.001$ in both cases). Using the GOLD 2008b staging definitions and the ECSC predicted FEV1, the obstructive individuals were classified as having mild $(17 ; 13.71 \%)$, moderate $(48 ; 38.71 \%)$, severe $(40 ; 32.26 \%)$ and very severe $(19 ; 15.32 \%)$ COPD. The corresponding figures for the same classification, based on our derived equations for FEV1, were 17 (13.71\%), 44 (35.48\%), $42(33.87 \%)$ and 21 (16.94\%), respectively. The overall agreement between the two classifications was $97.85 \%$ with the kappa coefficient of agreement indicating a very good agreement $(\mathrm{kappa}=0.936 ; \mathrm{p}<0.001)$. Out of the 124 obstructive individuals, 4 (3.2\%) were found to have an FEV1/ FVC ratio which was above the LLN as predicted from our equations.

Conclusion The statistical analysis has shown a high correlation between the parameters already used and those locally derived.

\section{Diagnosis and management of paediatric lung disease}

\section{P87 REPEAT SURVEY OF VITAMIN K PRESCRIBING PATTERNS AND BONE HEALTH SURVEILLANCE IN UK PAEDIATRIC CF CENTRES}

MR Nortier, DS Urquhart. Department of Paediatric Respiratory Medicine, Royal Hospital for Sick Children, Edinburgh, UK

10.1136/thoraxjnl-2015-207770.224

Introduction and objectives CF bone disease is multi-factorial; UK guidelines for screening and treatment of CF bone disease are published. ${ }^{1}$ Despite evidence of a key role for Vitamin $\mathrm{K}$ in bone formation, there is limited agreement on supplementation in CF. A previous 2005/06 survey ${ }^{2}$ of bone health surveillance and Vitamin $\mathrm{K}$ use in $\mathrm{CF}$ reported wide variation in practice. The current survey aimed to ascertain practice 10 years on. Methods Questionnaires were sent via email to all 25 UK paediatric CF centres. Data were collected on use of vitamins A, D, E and $\mathrm{K}$ including preparation, dose and criteria for Vitamin $\mathrm{K}$ supplementation. In addition, information was obtained on bone health surveillance including use of dual-energy X-ray absorptiometry (DXA) scanning to measure bone mineral density (BMD).

Results A 60\% questionnaire response representing 2805 CF children was collected. All centres reported that $>90 \%$ pancreatic insufficient patients receive multivitamin supplements and $12 / 15$ centres reported $>90 \%$ patients receive additional Vitamin E.

Only 3 centres routinely supplement Vitamin K, with only 1 reporting that $>90 \%$ patients receive Vitamin K. Criteria for prescribing Vitamin K were deranged liver function (10/15), clotting (5/15), low Vitamin K levels (2/15), and low BMD (3/15). Vitamin K dosage varied from $0.3-10 \mathrm{mg} /$ day, with most (12/15) prescribing $10 \mathrm{mg} /$ day. Menadiol was mainly (10/15) used with some using Phytomenadione for younger patients. Four centres used AquaDEKs, whilst three reported limitations in prescribing AquaDEKs due to formulary constraints. 